\title{
Multi-Resampling Doppler Compensation in Cooperative Underwater OFDM Systems
}

\author{
Bahattin Karakaya*, Mazen Omar Hasna*, Tolga M. Duman ${ }^{\dagger}$, Murat Uysal ${ }^{\ddagger}$ Ali Ghrayeb $^{\S}$ \\ *Dept. of Electrical Eng., Qatar University, Doha, Qatar, 2713 Email: \{bahattin,hasna\}@ qu.edu.qa \\ ${ }^{\dagger}$ Dept. of Electrical and Electronics Eng., Bilkent University, Ankara, Turkey, Email: duman@ee.bilkent.edu.tr \\ $\ddagger$ Dept. of Electrical and Electronics Eng., Özyeǧin University, Istanbul, Turkey, 34662 Email: murat.uysal@ozyegin.edu.tr \\ $\S$ Dept. of Electrical Eng., Texas A\&M Univ., Doha, Qatar, Email: ali.ghrayeb@qatar.tamu.edu
}

\begin{abstract}
We consider a multi-carrier cooperative underwater acoustic communication (UWAC) system and investigate the Doppler scaling problem arising due to the motion of different nodes. Specifically, we assume an orthogonal frequency division multiplexing (OFDM) system with amplify and forward (AF) relaying. Our channel model is built on large-scale path loss along with the short-term frequency-selective fading. For Doppler scaling compensation, we use multi-resampling (MR) receiver designs both at the relay and destination nodes. We present an extensive Monte Carlo simulation study to evaluate the error rate performance of the proposed UWAC system. In simulations, we use the publicly available VirTEX software in conjunction with the ray-tracing based $B E L L H O P$ software to precisely reflect the characteristics of an underwater geographical location and the movement of the nodes.
\end{abstract}

Index Terms - Underwater acoustic communications, amplify and forward relaying, orthogonal frequency division multiplexing, space time block coding, Doppler scaling, multi-resampling receiver.

\section{INTRODUCTION}

Cooperative diversity promises significant performance gains in terms of link reliability, spectral efficiency, system capacity, and transmission range [1]. Since underwater acoustic (UWA) channels experience frequency selectivity, singlecarrier modulations necessitate the use of equalizers which might be computationally prohibitive particularly for large channel lengths. Therefore, OFDM is considered as a strong candidate for deployment in UWAC systems because of its advantages in lessening the severe effects of frequency selective fading [2]. However, due to channel variations, in OFDM systems the orthogonality of the subcarriers may be destroyed and inter-carrier interference (ICI) may result. Moreover, usage of the cooperative diversity techniques may further complicate the mitigation of ICI.

In this paper, we focus on motion-induced Doppler distortion as the main source of ICI. Compared to radio frequency (RF) communications, small motion in underwater acoustic systems results in severe Doppler scaling effects as a result of the low speed of sound [3]. This scaling effect is exhibited as time compression or dilation of the signal, that is proportional to the Doppler scaling factor over the propagation path. These

This work is supported by Qatar National Research Fund (QNRF) grant through National Priority Research Program (NPRP) No. 09-242-2-099. QNRF is an initiative of Qatar Foundation. path-specific Doppler scaling factors can take values different from each other. In cooperative communication systems, in addition to the path-specific Doppler scaling factors, different mobile terminals have link-specific Doppler scaling factors because of their different relative speeds. Therefore the associated Doppler scaling factors for each tap and link may be significantly different from each other.

For Doppler compensation, resampling of the received signal with an estimate of the Doppler scaling factor can be utilized. We refer to this as the single-resampling (SR) method. The SR method is an optimal compensation algorithm for a scenario when all propagation paths are characterized with nearly identical Doppler scaling factors [4]. However, as discussed above, this assumption may not be accurate in practice. To improve the SR compensation for dissimilar Doppler scaling factors among the propagation paths, optimization of resampling rate for SR compensation is performed in [5]. A different approach is introduced for the resampling type compensation for the Doppler scaling problem in [6], named multi-resampling (MR) method. The MR method outperforms the SR method for both path and user specific Doppler distortions. In practical systems, for both SR and MR methods, it may not be possible to acquire a fully ICI free transmission. Hence, after resampling, other ICI mitigation techniques may be utilized to take care of the residual effects [7].

There are sporadic works on cooperative communications using OFDM for the Doppler scaling problem in UWAC systems [8], [9]. In this paper, we consider path-specific and link-specific Doppler distortions together in multi-carrier cooperative UWAC system. We consider an aggregate channel model that consists of large-scale path loss, small-scale fading and compression/dilation effects. Particularly, for the path loss analysis, we use the BELLHOP software; for Doppler distortion modeling, we use the VirTEX software which precisely reflects the characteristics of a geographical location (such as sound speed profile, sound frequency, bathymetry, the type of bottom sediments, depths of nodes, etc.) and motion of terminals [10], [11]. To mitigate the effects of ICI at the receiver terminals, MR method is used at the receiver frontends of the relay and destination nodes.

The rest of the paper is organized as follows. In Section II, we provide the point to point system and channel models. 


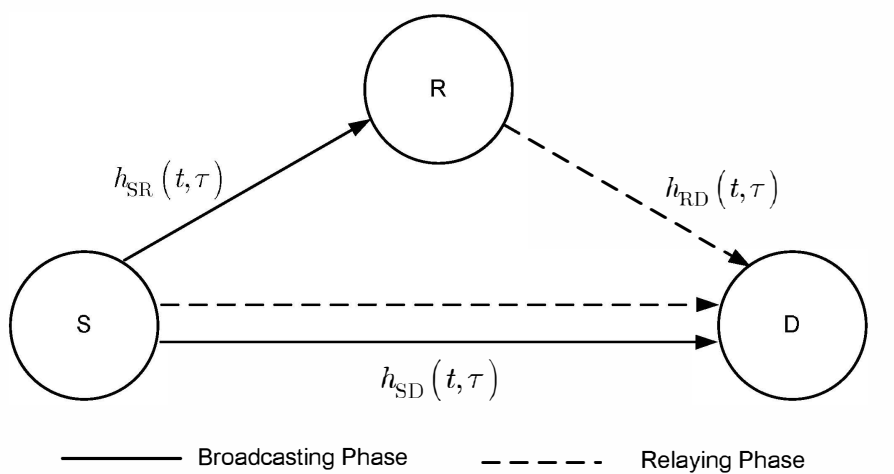

Fig. 1. Schematic representation of relay-assisted Transmit Diversity Cooperation.

Also, we present the MR receiver front-ends for the space time block coded (STBC) cooperative OFDM system under consideration. Moreover, we derive the input output expressions and noise cross correlations for data detection. In Section III, we present our simulation results and provide several insights into UWAC system performance. Finally, in Section IV, we conclude the paper.

Notation: $\operatorname{Tr}(\mathbf{A})$ denotes trace of matrix A. $(.)^{*},(.)^{T}$ and, $(.)^{H}$ denote conjugate, transpose, and Hermitian transpose operations, respectively. $\operatorname{Re}\{\cdot\}$ denotes the real part. $\operatorname{Dec}\{$. denotes the symbol decision. $\mathbf{0}_{N}$ denotes $N \times N$ and $\mathbf{0}_{M \times N}$ denotes $M \times N$ zero matrices. Bold upper-case letters denote matrices and bold lower-case letters denote vectors. $\mathbf{a}^{\# b}$ denotes a discrete signal vector a during the $b^{\text {th }}$ tranmission block.

\section{SYSTEM MODEL}

We consider a cooperative UWAC system in which the source node (S) communicates to the destination node (D) with the assistance of a single-relay (R), see Fig. 1. All terminals are half duplex and equipped with a single transducer and hydrophone pair. Specifically, we assume an OFDM system with $\mathrm{AF}$ relaying in which the relay terminal resamples, whitens, amplifies and retransmits the received signal from the source terminal in the first signaling interval. To achieve full transmit diversity, STBC is used with the time diversity (TD) relaying protocol of [1]. The required four transmission blocks and the coded data transmission over source and relay nodes are shown in Fig. 2. We assume that the channel path gains, path delays and Doppler scaling factors of the links are constant over four consecutive blocks. In the following subsection, the mathematical model of the point-to-point transmission is established. In the second subsection, the received signals for both broadcasting phase and relaying phase are presented. Moreover, received data expressions are derived at the output of MR resampling blocks for the proposed cooperative system. Required cross correlations of noise terms are provided in the third subsection.

\begin{tabular}{|l|c|c|c|c|c|c|c|c|}
\hline $\mathrm{CP}$ & $\mathbf{0}_{K}$ & $\mathrm{CP}$ & $\mathbf{d}_{1}$ & $\mathrm{CP}$ & $\mathbf{0}_{K}$ & $\mathrm{CP}$ & $-\mathbf{d}_{2}^{*}$ & $\mathrm{R} \rightarrow \mathrm{D}$ \\
\hline & Block 1 & Block 2 & Block 3 & Block 4 & \\
\hline $\mathrm{CP}$ & $\mathbf{d}_{1}$ & $\mathrm{CP}$ & $\mathbf{d}_{2}$ & $\mathrm{CP}$ & $-\mathbf{d}_{2}^{*}$ & $\mathrm{CP}$ & $\mathbf{d}_{1}^{*}$ & $\mathrm{~S} \rightarrow \mathrm{D}$ \\
\hline
\end{tabular}

Fig. 2. Transmission block format for TD-STBC.

\section{A. Point-to-Point Transmission}

Let $T$ denote the OFDM symbol duration, $T_{C P}$ the cyclic prefix duration and $T_{P}$ the postfix duration. Throughout this work, we assume that the $T_{C P}$ is sufficiently long to prevent inter- block interference. The postfix is added to avoid transient interference in resampling. The total OFDM block duration is $T^{\prime}=T+T_{C P}+T_{P}$. The frequency spacing is $\Delta f=1 / T$. The $k$ th subcarrier is at the frequency

$$
f_{k}=f_{0}+k \Delta f, \quad k=0, \ldots, K-1,
$$

where $f_{0}$ is the lowest subcarrier frequency in the passband and $K$ subcarriers are used, so the bandwidth is $B=K \Delta f$. For the sake of presentation, instead of describing each link (i.e. $\mathrm{S} \rightarrow \mathrm{R}, \mathrm{R} \rightarrow \mathrm{D}$ and $\mathrm{S} \rightarrow \mathrm{D}$ ) separately, transmit node is denoted by $\mathrm{X}$ and receive node is denoted by $\mathrm{Y}$. The passband transmitted signal is given by

$$
\tilde{s}_{\mathbf{X}}(t)=\sqrt{\varepsilon_{\mathbf{X}}} \operatorname{Re}\left\{\left[\sum_{k=0}^{K-1} d(k) e^{j 2 \pi k \Delta f t}\right] e^{j 2 \pi f_{0} t} g(t)\right\},
$$

where $\varepsilon_{\mathrm{X}}$ denotes the power of baseband equivalent transmitted signal at the node $\mathrm{X}$, and $\{d(k)\}_{k=0}^{K-1}$ denote the BPSK modulated symbols. $g(t)=1 / \sqrt{T}$ is a rectangular pulse of duration $T^{\prime}$. The impulse response of $\mathrm{X} \rightarrow \mathrm{Y}$ link is given by

$$
h_{\mathrm{XY}}(t, \tau)=\sqrt{G_{\mathrm{XY}}} \sum_{c=0}^{N_{c}^{\mathrm{XY}}-1} \sum_{p=0}^{N_{p}^{(c) \mathrm{XY}}-1} h_{c, p}^{\mathrm{XY}} \delta\left(\tau-\tau_{c, p}^{\mathrm{XY}}(t)\right) \text {, }
$$

where $h_{c, p}^{\mathrm{XY}}$ is the path amplitude, $\tau_{c, p}^{\mathrm{XY}}(t)$ is the time varying path delay and $G_{\mathrm{XY}}$ is the path loss coefficient for the $\mathrm{X} \rightarrow \mathrm{Y}$ link. $N_{c}^{\mathrm{XY}}$ is the number of clusters where each cluster has $N_{p}^{(c) X Y}$ paths and all paths in the same cluster has a common Doppler scaling factor given by

$$
\tau_{c, p}^{\mathrm{XY}}(t)=\tau_{c, p}^{\mathrm{XY}}-a_{c}^{\mathrm{XY}} t .
$$

We assume that power delay profile of the channel is normalized such that

$$
\varepsilon_{\mathrm{XY}}=\sum_{c=0}^{N_{c}^{\mathrm{XY}}-1} \sum_{p=0}^{N_{p}^{(c) \mathrm{XY}}-1}\left|h_{c, p}^{\mathrm{XY}}\right|^{2}=1 .
$$


$\tilde{v}_{\mathrm{Y}}(t)=\sqrt{E_{\mathrm{XY}}} R e\left\{\sum_{c=0}^{N_{c}^{\mathrm{XY}}-1} \sum_{p=0}^{N_{p}^{(c) \mathrm{XY}}-1} h_{c, p}^{\mathrm{XY}}\left[\sum_{k=0}^{K-1} d(k) e^{j 2 \pi k \Delta f\left(t+a_{c}^{\mathrm{XY}} t-\tau_{c, p}^{\mathrm{XY}}\right)} g\left(t+a_{c}^{\mathrm{XY}} t-\tau_{c, p}^{\mathrm{XY}}\right)\right] e^{j 2 \pi f_{0}\left(t+a_{c}^{\mathrm{XY}} t-\tau_{c, p}^{\mathrm{XY}}\right)}\right\}+\tilde{w}_{\mathrm{Y}}(t)$

The passband received signal can be written as in (6) (shown at the top of the page) $E_{\mathrm{XY}}=\varepsilon_{\mathrm{X}} G_{\mathrm{XY}}$ denotes the received energy of baseband equivalent received signal at node $\mathrm{Y}$, $\tilde{w}(t)$ is a white Gaussian noise (WGN) process with power spectral density (PSD) $N_{0} / 2$. The baseband version $v_{\mathrm{Y}}(t)$ of the received signal satisfies $\tilde{v}_{\mathrm{Y}}(t)=\operatorname{Re}\left\{v_{\mathrm{Y}}(t) e^{j 2 \pi f_{\bullet} t}\right\}$, and can be written as

$$
v_{\mathrm{Y}}(t)=\sqrt{E_{\mathrm{XY}}} \sum_{k=0}^{K-1} d(k) P_{k}^{\mathrm{XY}}(t)+w_{\mathrm{Y}}(t)
$$

where $w_{\mathrm{Y}}(t)$ is a complex WGN with PSD $N_{0}$,

$$
\begin{aligned}
P_{k}^{\mathrm{XY}}(t)= & \sum_{c=0}^{N_{c}^{\mathrm{XY}}-1} \sum_{p=0}^{N_{c}^{(c), \mathrm{XY}}-1} \alpha_{c, p}^{\mathrm{XY}}(k) e^{j 2 \pi k \Delta f t} e^{j 2 \pi a_{c}^{\mathrm{XY}} f_{k} t} \\
& \times g\left(t+a_{c}^{\mathrm{XY}} t-\tau_{c}^{\mathrm{XY}}\right)
\end{aligned}
$$

and

$$
\alpha_{c, p}^{\mathrm{XY}}(k)=h_{c, p}^{\mathrm{XY}} e^{-j 2 \pi f_{k} \tau_{c, p}^{\mathrm{XY}}} .
$$

We assume perfect channel state information (CSI) at the receiver. This interpretation allows us to apply the classical maximum-likelihood detection theory (see [6]). First, we notice that a set of sufficient statistics is given by the outputs of a bank of filters matched to the pulses modulating the symbols. Namely, the samples

$$
r_{\mathrm{Y}}(k)=\int_{-\infty}^{\infty} v_{\mathrm{XY}}(t) P_{k}^{\mathrm{XY} *}(t) d t, \quad k=0,1, \ldots, K-1,
$$

are sufficient statistics for maximum-likelihood detection. The relevant discrete channel model yields

$$
r_{\mathrm{Y}}(k)=\sqrt{E_{\mathrm{XY}}} \sum_{l=0}^{K-1} H_{k, l}^{\mathrm{XY}} d(l)+n_{\mathrm{Y}}(k),
$$

where

$$
H_{k, l}^{\mathrm{XY}}=\int_{-\infty}^{\infty} P_{l}^{\mathrm{XY}}(t) P_{k}^{\mathrm{XY}}(t)^{*} d t .
$$

In (11), $n_{\mathrm{Y}}(k)$ is an additive Gaussian noise term with a crosscorrelation of [12]

$$
\Omega_{k, l}=E\left[n_{\mathrm{Y}}(k) n_{\mathrm{Y}}(l)^{*}\right] \approx N_{0} H_{k, l}^{\mathrm{XY}} .
$$

Equation (11) can be written in a matrix form as,

$$
\mathbf{r}_{\mathrm{Y}}=\sqrt{E_{\mathrm{XY}}} \mathbf{H}_{\mathrm{XY}} \mathbf{d}+\mathbf{n}_{\mathrm{Y}},
$$

where $\left\{\mathbf{H}_{\mathrm{XY}}\right\}_{k, l}=H_{k, l}^{\mathrm{XY}}, \mathbf{r}_{\mathrm{Y}}=\left[r_{\mathrm{Y}}(0), \ldots, r_{\mathrm{Y}}(K-1)\right]^{T}$ and, $\mathbf{n}_{\mathrm{Y}}=\left[n_{\mathrm{Y}}(0), \ldots, n_{\mathrm{Y}}(K-1)\right]^{T}$.
We now discuss the implementation of the matched filtering in (10). Let us manipulate (10) as follows

$$
r_{\mathrm{Y}}(k) \approx \sum_{c=\mathbf{0}}^{N_{c}^{\mathrm{XY}}-1} \alpha^{(c) \mathrm{XY}}(k)^{*} \int_{\mathbf{0}}^{T} v_{\mathrm{Y}}^{(c) \mathrm{XY}}\left(\frac{\xi}{1+a_{c}^{\mathrm{XY}}}\right) e^{-j 2 \pi k \xi / T} d \xi,
$$

where

$$
\alpha^{(c) \mathrm{XY}}(k)=\sum_{p=0}^{N_{p}^{(c) \mathrm{XY}}-1} \frac{\alpha_{p}^{(c) \mathrm{XY}}(k)}{\sqrt{T}\left(1+a_{c}^{\mathrm{XY}}\right)},
$$

and

$$
v_{\mathrm{Y}}^{(c) \mathrm{XY}}(t)=v_{\mathrm{Y}}(t) e^{-j 2 \pi t a_{c}^{\mathrm{XY}} f_{\bullet}} .
$$

In (15), the integration can be easily performed using FFT. (17) corresponds to Doppler induced frequency shift compensation. Changing variable $t$ of (17) to $\frac{\xi}{1+a_{\mathrm{XY}}}$ in (15) corresponds to resampling. These implementation details are shown in detail in Fig. 3 for $\mathrm{S} \rightarrow \mathrm{R}$ link Doppler compensation. MR resampling compensation blocks are shown as boxes at the destination for broadcasting and relaying phases in Fig. 3 and Fig. 4, respectively.

\section{B. Multiple-Resampling Based Receiver Front-End}

In this subsection, we provide MR resampling front-end outputs at receive nodes step by step. In the considered TD protocol of AF relaying, source terminal communicates with the relay and destination terminals during the first signaling interval (broadcasting phase). The baseband received signal in the first transmission block at the destination node can be written from (7) as

$$
v_{\mathrm{D}}^{\# 1}(t)=\sqrt{E_{\mathrm{SD}}} \sum_{k=0}^{K-1} d_{1}(k) P_{k}^{\mathrm{SD}}(t)+w_{\mathrm{D}}^{\# 1}(t) .
$$

The received data after resampling is obtained from (14) as

$$
\mathbf{r}_{\mathrm{D}}^{\# 1}=\sqrt{E_{\mathrm{SD}}} \mathbf{H}_{\mathrm{SD}} \mathbf{d}_{1}+\mathbf{n}_{\mathrm{D}}^{\# 1} .
$$

The relay terminal compensates the Doppler distortion, whitens and amplifies the signal before transmitting the received signal to the destination, see Fig. 3. The baseband received signal in the first transmission block at the relay node can be written from (7) as

$$
v_{\mathrm{R}}^{\# 1}(t)=\sqrt{E_{\mathrm{SR}}} \sum_{k=0}^{K-1} d_{1}(k) P_{k}^{\mathrm{SR}}(t)+w_{\mathrm{R}}^{\# 1}(t) .
$$

Similar to destination node, the received data after resampling is given by

$$
\mathrm{r}_{\mathrm{R}}^{\# 1}=\sqrt{\mathbf{E}_{\mathrm{SR}}} \mathbf{H}_{\mathrm{SR}} \mathbf{d}_{\mathbf{1}}+\mathbf{n}_{\mathrm{R}}^{\# \mathbf{1}} .
$$




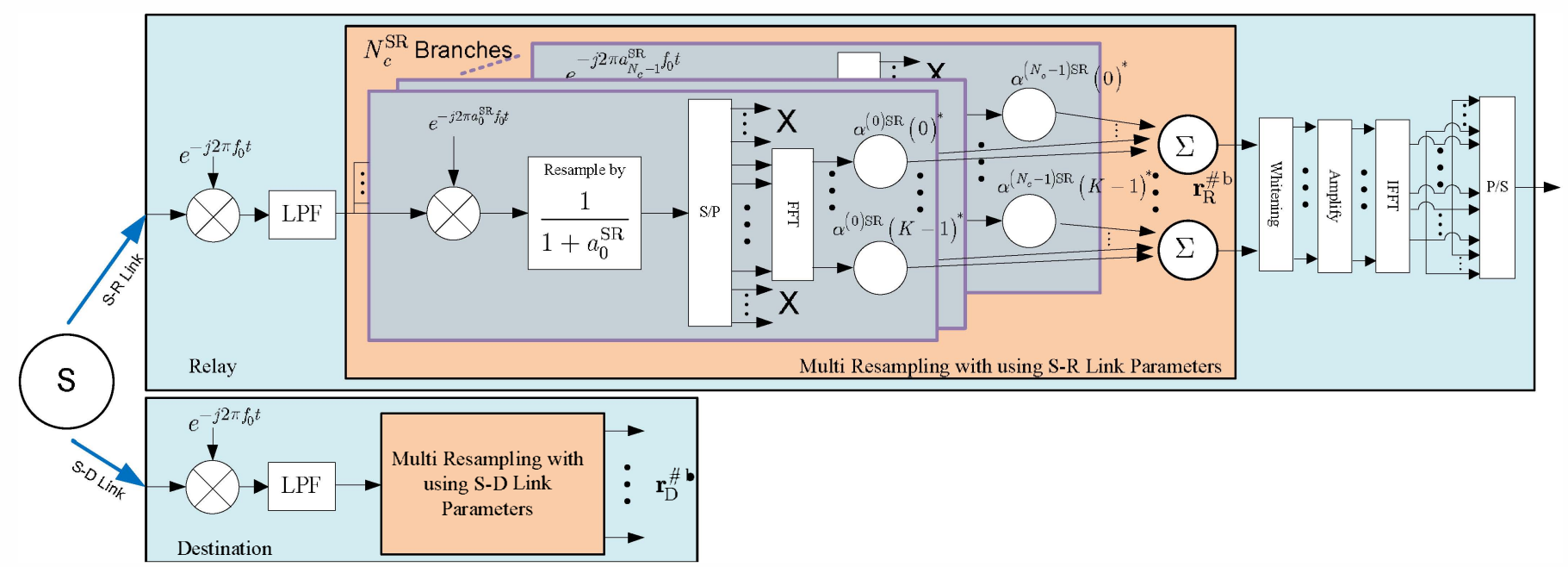

Fig. 3. Relay and destination terminal receiver front-ends for the broadcasting phase.

Here the noise is not white anymore and its cross-correlation can be written from (13) as

$$
\boldsymbol{\Omega}_{\mathrm{SR}}=E\left[\mathbf{n}_{\mathrm{R}}^{\# 1} \mathbf{n}_{\mathrm{R}}^{\# 1}\right] \approx N_{0} \mathbf{H}_{\mathrm{SR}} .
$$

For the whitening process, we use the square root of the matrix $\mathbf{H}_{\mathrm{SR}}$ as

$$
\tilde{\mathbf{r}}_{\mathrm{R}}^{\# 1}=\left(\mathbf{H}_{\mathrm{SR}}\right)^{-1 / 2} \mathbf{r}_{\mathrm{R}}^{\# 1}
$$

and we obtain

$$
\tilde{\mathbf{r}}_{\mathrm{R}}^{\# 1}=\sqrt{E_{\mathrm{SR}}} \tilde{\mathbf{H}}_{\mathrm{SR}} \mathbf{d}_{1}+\tilde{\mathbf{n}}_{\mathrm{R}}^{\# 1}
$$

where

$$
\tilde{\mathbf{H}}_{\mathrm{SR}}=\left(\mathbf{H}_{\mathrm{SR}}\right)^{-1 / 2} \mathbf{H}_{\mathrm{SR}} .
$$

The average received signal energy at the receiver after whitening is given by

$$
\varepsilon_{a m p}=E_{\mathrm{SR}}+N_{0} .
$$

Hence, the amplified signal in frequency domain at the relay can be written as

$$
\overline{\mathbf{r}}_{\mathrm{R}}^{\# 1}=\frac{\tilde{\mathbf{r}}_{\mathrm{R}}^{\# 1}}{\sqrt{\varepsilon_{a m p}}} .
$$

In the second signaling interval (relaying phase), both the relay and source terminals communicate with the destination terminal. In the broadcasting phase, the destination receiver is concerned about only SD link parameters, however, in the relaying phase it is concerned with both SD and RD link parameters, see Fig. 4. The baseband received signal at the destination for the second transmission block can be written as

$$
\begin{aligned}
v_{\mathrm{D}}^{\# 2}(t)= & \sqrt{E_{\mathrm{RD}}} \sum_{k=0}^{K-1} \bar{r}_{\mathrm{SR}}^{\# 1}(k) P_{k}^{\mathrm{RD}}(t) \\
& +\sqrt{E_{\mathrm{SD}}} \sum_{k=0}^{K-1} d_{2}(k) P_{k}^{\mathrm{SD}}(t)+w_{\mathrm{D}}^{\# 2}(t),
\end{aligned}
$$

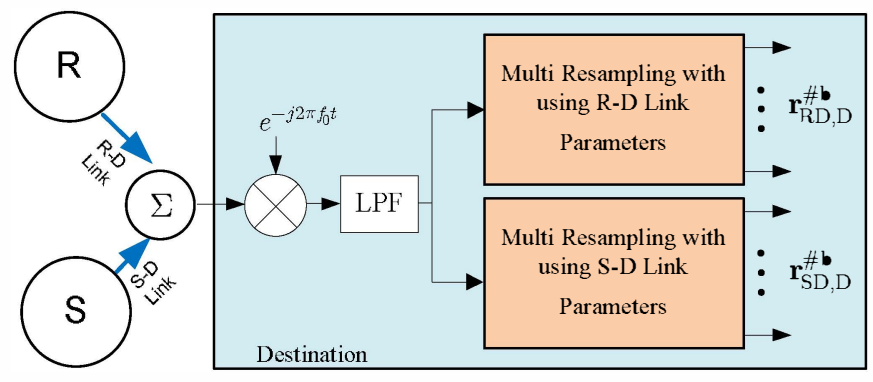

Fig. 4. Destination terminal receiver front-end for the relaying phase.

where $w_{\mathrm{D}}^{\# 2}(t)$ is a complex WGN with PSD $N_{0}$. In this case, at the receiver the number of resampling branches is multiplied by the number of transmitters to cope with the ICI effects of different Doppler scaling factors [6]. The output of the first main branch can be found by matched filtering the received signal by $\mathrm{R} \rightarrow \mathrm{D}$ link parameters as

$$
r_{\mathrm{RD}, \mathrm{D}}^{\# 2}(k)=\int_{-\infty}^{\infty} v_{D}^{\# 2}(t) P_{k}^{\mathrm{RD}}(t)^{*} d t, \quad k=0,1, \ldots, K-1 .
$$

This yields

$$
\begin{aligned}
r_{\mathrm{RD}, \mathrm{D}}^{\# 2}(k) & =\sqrt{E_{\mathrm{RD}}} \sum_{l=0}^{K-1} H_{k, l}^{\mathrm{RD}} \bar{r}_{\mathrm{R}}^{\# 1}(l) \\
& +\sqrt{E_{\mathrm{SD}}} \sum_{l=0}^{K-1} H_{k, l}^{\mathrm{RD}, \mathrm{SD}} d_{2}(l)+n_{\mathrm{RD}, \mathrm{D}}^{\# 2}(k),
\end{aligned}
$$

where

$$
H_{k, l}^{\mathrm{RD}}=\int_{-\infty}^{\infty} P_{l}^{\mathrm{RD}}(t) P_{k}^{\mathrm{RD}}(t)^{*} d t
$$

and

$$
H_{k, l}^{\mathrm{RD}, \mathrm{SD}}=\int_{-\infty}^{\infty} P_{l}^{\mathrm{SD}}(t) P_{k}^{\mathrm{RD}}(t)^{*} d t
$$


Equation (30) can be written in matrix form as

$\mathbf{r}_{\mathrm{RD}, \mathrm{D}}^{\# 2}=\sqrt{E_{\mathrm{RD}}} \mathbf{H}_{\mathrm{RD}} \overline{\mathbf{r}}_{\mathrm{SR}}^{\# 1}+\sqrt{E_{\mathrm{SD}}} \mathbf{H}_{\mathrm{RD}, \mathrm{SD}} \mathbf{d}_{2}+\mathbf{n}_{\mathrm{RD}, \mathrm{D}}^{\# 2}$

By substituting (27) into (33), we obtain

$$
\begin{aligned}
\mathbf{r}_{\mathrm{RD}, \mathrm{D}}^{\# 2}= & \sqrt{\frac{E_{\mathrm{SR}} E_{\mathrm{RD}}}{\varepsilon_{a m p}}} \mathbf{H}_{\mathrm{RD}} \tilde{\mathbf{H}}_{\mathrm{SR}} \mathbf{d}_{1}+\sqrt{E_{\mathrm{SD}}} \mathbf{H}_{\mathrm{RD}, \mathrm{SD}} \mathbf{d}_{2} \\
& +\sqrt{\frac{E_{\mathrm{RD}}}{\varepsilon_{a m p}}} \mathbf{H}_{\mathrm{RD}} \mathbf{n}_{\mathrm{R}}^{\# 1}+\mathbf{n}_{\mathrm{RD}, \mathrm{D}}^{\# 2}
\end{aligned}
$$

Similar to the first main branch output, the second main branch output can be found by matched filtering the received signal by $\mathrm{S} \rightarrow \mathrm{D}$ link parameters as [6]

$$
r_{\mathrm{SD}, \mathrm{D}}^{\# 2}(k)=\int_{-\infty}^{\infty} v_{D}^{\# 2}(t) P_{k}^{\mathrm{SD}}(t)^{*} d t, \quad k=0,1, \ldots, K-1,
$$

which gives

$$
\begin{aligned}
r_{\mathrm{SD}, \mathrm{D}}^{\# 2}(k)= & \sqrt{E_{\mathrm{RD}}} \sum_{l=0}^{K-1} H_{k, l}^{\mathrm{SD}, \mathrm{RD}} \bar{r}_{\mathrm{R}}^{\# 1}(l) \\
& +\sqrt{E_{\mathrm{SD}}} \sum_{l=0}^{K-1} H_{k, l}^{\mathrm{SD}} d_{2}(l)+n_{\mathrm{SD}, \mathrm{D}}^{\# 2}(k),
\end{aligned}
$$

where

$$
H_{k, l}^{\mathrm{SD}}=\int_{-\infty}^{\infty} P_{l}^{\mathrm{SD}}(t) P_{k}^{\mathrm{SD}}(t)^{*} d t
$$

and

$$
H_{k, l}^{\mathrm{SD}, \mathrm{RD}}=\int_{-\infty}^{\infty} P_{l}^{\mathrm{RD}}(t) P_{k}^{\mathrm{SD}}(t)^{*} d t .
$$

The above equation can be written in a matrix form as

$$
\mathbf{r}_{\mathrm{SD}, \mathrm{D}}^{\# 2}=\sqrt{E_{\mathrm{RD}}} \mathbf{H}_{\mathrm{SD}, \mathrm{RD}} \overline{\mathbf{r}}_{\mathrm{SR}}^{\# 1}+\sqrt{E_{\mathrm{SD}}} \mathbf{H}_{\mathrm{SD}} \mathbf{d}_{2}+\mathbf{n}_{\mathrm{SD}, \mathrm{D}}^{\# 2}
$$

By substituting (27) into (39), we obtain

$$
\begin{aligned}
\mathbf{r}_{\mathrm{SD}, \mathrm{D}}^{\# 2}= & \sqrt{\frac{E_{\mathrm{SR}} E_{\mathrm{RD}}}{\varepsilon_{a m p}}} \mathbf{H}_{\mathrm{SD}, \mathrm{RD}} \tilde{\mathbf{H}}_{\mathrm{SR}} \mathbf{d}_{1}+\sqrt{E_{\mathrm{SD}}} \mathbf{H}_{\mathrm{SD}} \mathbf{d}_{2} \\
& +\sqrt{\frac{E_{\mathrm{RD}}}{\varepsilon_{a m p}}} \mathbf{H}_{\mathrm{SD}, \mathrm{RD}} \mathbf{n}_{\mathrm{R}}^{\# 1}+\mathbf{n}_{\mathrm{SD}, \mathrm{D}}^{\# 2}
\end{aligned}
$$

Since STBC is used in the cooperation protocol under consideration, a total of four signaling time blocks are needed. For the third and fourth transmission blocks, similar to equations (19), (34) and (40), the input-output relations can be written in matrix form as

$$
\begin{aligned}
& \mathbf{r}_{\mathrm{D}}^{\# 3}=-\sqrt{E_{\mathrm{SD}}} \mathbf{H}_{\mathrm{SD}} \mathbf{d}_{2}^{*}+\mathbf{n}_{\mathrm{D}}^{\# 3} \\
\mathbf{r}_{\mathrm{RD}, \mathrm{D}}^{\# 4}= & -\sqrt{\frac{E_{\mathrm{SR}} E_{\mathrm{RD}}}{\varepsilon_{a m p}}} \mathbf{H}_{\mathrm{RD}} \tilde{\mathbf{H}}_{\mathrm{SR}} \mathbf{d}_{2}^{*}+\sqrt{E_{\mathrm{SD}}} \mathbf{H}_{\mathrm{RD}, \mathrm{SD}} \mathbf{d}_{1}^{*} \\
& +\sqrt{\frac{E_{\mathrm{RD}}}{\varepsilon_{a m p}}} \mathbf{H}_{\mathrm{RD}} \mathbf{n}_{\mathrm{R}}^{\# 3}+\mathbf{n}_{\mathrm{RD}, \mathrm{D}}^{\# 4}
\end{aligned}
$$

and

$$
\begin{aligned}
\mathbf{r}_{\mathrm{SD}, \mathrm{D}}^{\# 4}= & -\sqrt{\frac{E_{\mathrm{SR}} E_{\mathrm{RD}}}{\varepsilon_{a m p}}} \mathbf{H}_{\mathrm{SD}, \mathrm{RD}} \tilde{\mathbf{H}}_{\mathrm{SR}} \mathbf{d}_{2}^{*}+\sqrt{E_{\mathrm{SD}}} \mathbf{H}_{\mathrm{SD}} \mathbf{d}_{1}^{*} \\
& +\sqrt{\frac{E_{\mathrm{RD}}}{\varepsilon_{a m p}}} \mathbf{H}_{\mathrm{SD}, \mathrm{RD}} \mathbf{n}_{\mathrm{R}}^{\# 3}+\mathbf{n}_{\mathrm{SD}, \mathrm{D}}^{\# 4}
\end{aligned}
$$

All input output equations can be collected in one equation as given by (44) (at the top of the next page).

In the following subsection, cross correlations of resulting additive noise terms of this four block time duration STBC coded TD relaying system are provided. We will use this system model and colored noise statistics in conjunction with minimum mean square error (MMSE) estimation to detect the transmitted symbols.

\section{Cross-Correlation Properties of the Noise Terms}

Cross correlation of the noise vector can be constructed with block matrices as

$\Omega=E\left\{\mathbf{n n}^{H}\right\}=\left[\begin{array}{cccc}\Omega_{1} & \mathbf{0}_{K \times 2 K} & \multicolumn{2}{c}{\mathbf{0}_{3 K}} \\ \mathbf{0}_{2 K \times K} & \Omega_{2} & \Omega_{3} & \mathbf{0}_{K \times 2 K} \\ & \mathbf{0}_{3 K} & \mathbf{0}_{2 K \times K} & \Omega_{4}\end{array}\right]$ $(45)$

where the first and third block noise cross correlation matrices are given by using (13)

$$
\begin{gathered}
\Omega_{1}=E\left\{\mathbf{n}_{\mathrm{D}}^{\# 1}\left(\mathbf{n}_{\mathrm{D}}^{\# 1}\right)^{H}\right\}=N_{0} \mathbf{H}_{\mathrm{SD}}, \\
\Omega_{3}=E\left\{\left(\mathbf{n}_{\mathrm{D}}^{\# 3}\right)^{*}\left(\mathbf{n}_{\mathrm{D}}^{\# 3}\right)^{T}\right\}=N_{0} \mathbf{H}_{\mathrm{SD}}^{H} .
\end{gathered}
$$

In the second block, cross correlation matrix can be calculated as

$$
\begin{aligned}
& \Omega_{2}=\frac{E_{\mathrm{RD}}}{\varepsilon_{a m p}}\left[\begin{array}{c}
\mathbf{H}_{\mathrm{RD}} \\
\mathbf{H}_{\mathrm{SD}, \mathrm{RD}}
\end{array}\right] E\left\{\tilde{\mathbf{n}}_{\mathrm{R}}^{\# 1}\left(\tilde{\mathbf{n}}_{\mathrm{R}}^{\# 1}\right)^{H}\right\}\left[\begin{array}{ll}
\mathbf{H}_{\mathrm{RD}}^{H} & \mathbf{H}_{\mathrm{SD}, \mathrm{RD}}^{H}
\end{array}\right] \\
& +E\left\{\left[\begin{array}{cc}
\mathbf{n}_{\mathrm{RD}, \mathrm{D}}^{\# 2}\left(\mathbf{n}_{\mathrm{RD}, \mathrm{D}}^{\# 2}\right)^{H} & \mathbf{n}_{\mathrm{RD}, \mathrm{D}}^{\# 2}\left(\mathbf{n}_{\mathrm{SD}, \mathrm{D}}^{\# 2}\right)^{H} \\
\mathbf{n}_{\mathrm{SD}, \mathrm{D}}^{\# 2}\left(\mathbf{n}_{\mathrm{RD}, \mathrm{D}}^{\# 2}\right)^{H} & \mathbf{n}_{\mathrm{SD}, \mathrm{D}}^{\# 2}\left(\mathbf{n}_{\mathrm{SD}, \mathrm{D}}^{\# 2}\right)^{H}
\end{array}\right]\right\} \\
& =N_{0}\left(\frac{E_{\mathrm{RD}}}{\varepsilon_{a m p}}\left[\begin{array}{cc}
\mathbf{H}_{\mathrm{RD}} \mathbf{H}_{\mathrm{RD}}^{H} & \mathbf{H}_{\mathrm{RD}} \mathbf{H}_{\mathrm{SD}, \mathrm{RD}}^{H} \\
\mathbf{H}_{\mathrm{SD}, \mathrm{RD}} \mathbf{H}_{\mathrm{RD}}^{H} & \mathbf{H}_{\mathrm{SD}, \mathrm{RD}} \mathbf{H}_{\mathrm{SD}, \mathrm{RD}}^{H}
\end{array}\right]\right. \\
& \left.+\left[\begin{array}{cc}
\mathbf{H}_{\mathrm{RD}} & \mathbf{H}_{\mathrm{RD}, \mathrm{SD}} \\
\mathbf{H}_{\mathrm{SD}, \mathrm{RD}} & \mathbf{H}_{\mathrm{SD}}
\end{array}\right]\right) \text {. }
\end{aligned}
$$

In the fourth block, noise cross correlation matrix can be computed as

$$
\begin{aligned}
& \Omega_{4}=\frac{E_{\mathrm{RD}}}{\varepsilon_{a m p}}\left[\begin{array}{c}
\mathbf{H}_{\mathrm{RD}}^{*} \\
\mathbf{H}_{\mathrm{SD}, \mathrm{RD}}^{*}
\end{array}\right] E\left\{\left(\mathbf{n}_{\mathrm{R}}^{\# 3}\right)^{*}\left(\mathbf{n}_{\mathrm{R}}^{\# 3}\right)^{T}\right\}\left[\begin{array}{ll}
\mathbf{H}_{\mathrm{RD}}^{T} & \mathbf{H}_{\mathrm{SD}, \mathrm{RD}}^{T}
\end{array}\right] \\
& \left.+E\left\{\begin{array}{ll}
\left(\mathbf{n}_{\mathrm{RD}, \mathrm{D}}^{\# 4}\right)^{*}\left(\mathbf{n}_{\mathrm{RD}, \mathrm{D}}^{\# 4}\right)^{T} & \left(\mathbf{n}_{\mathrm{RD}, \mathrm{D}}^{\# 4}\right)^{*}\left(\mathbf{n}_{\mathrm{SD}, \mathrm{D}}^{\# 4}\right)^{T} \\
\left(\mathbf{n}_{\mathrm{SD}, \mathrm{D}}^{\# 4}\right)^{*}\left(\mathbf{n}_{\mathrm{RD}, \mathrm{D}}^{\# 4}\right)^{T} & \left(\mathbf{n}_{\mathrm{SD}, \mathrm{D}}^{\# 4}\right)^{*}\left(\mathbf{n}_{\mathrm{SD}, \mathrm{D}}^{\# 4}\right)^{T}
\end{array}\right]\right\} \\
& =N_{0}\left(\frac{E_{\mathrm{RD}}}{\varepsilon_{a m p}}\left[\begin{array}{cc}
\mathbf{H}_{\mathrm{RD}}^{*} \mathbf{H}_{\mathrm{RD}}^{T} & \mathbf{H}_{\mathrm{RD}}^{*} \mathbf{H}_{\mathrm{SD}, \mathrm{RD}}^{T} \\
\mathbf{H}_{\mathrm{SD}, \mathrm{RD}}^{*} \mathbf{H}_{\mathrm{RD}}^{T} & \mathbf{H}_{\mathrm{SD}, \mathrm{RD}}^{*} \mathbf{H}_{\mathrm{SD}, \mathrm{RD}}^{T}
\end{array}\right]\right. \\
& \left.+\left[\begin{array}{cc}
\mathbf{H}_{\mathrm{RD}} & \mathbf{H}_{\mathrm{RD}, \mathrm{SD}} \\
\mathbf{H}_{\mathrm{SD}, \mathrm{RD}} & \mathbf{H}_{\mathrm{SD}}
\end{array}\right]\right) \text {. }
\end{aligned}
$$




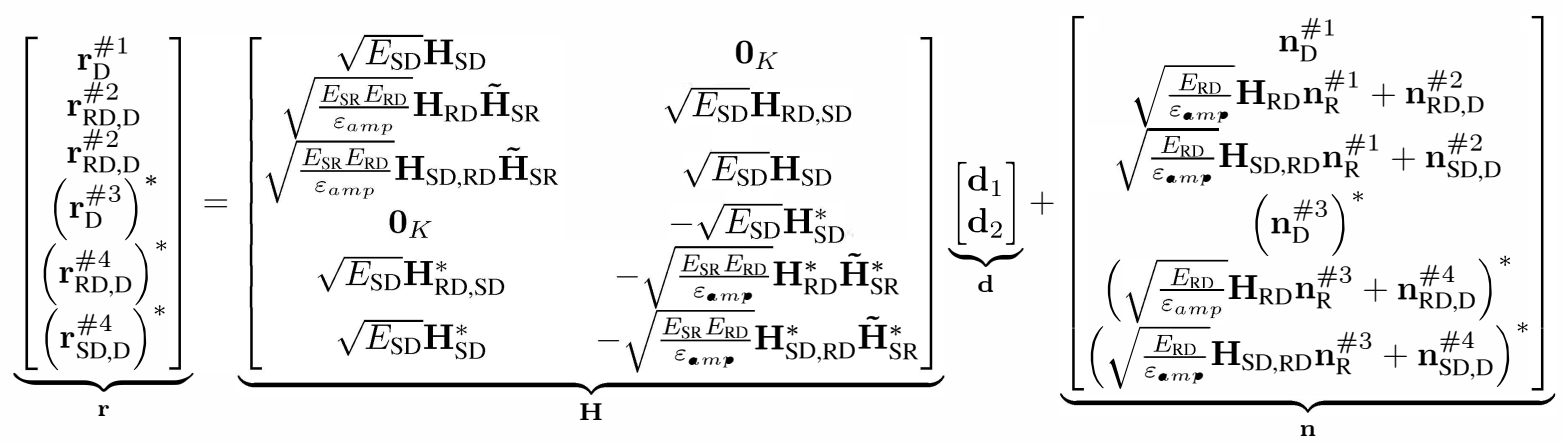

With given (44) and (45), transmitted symbols are estimated by using MMSE detector as follows

$$
\hat{\mathbf{d}}_{\mathrm{MMSE}}=\operatorname{Dec}\left\{\mathbf{H}^{H}\left(\mathbf{H} \mathbf{H}^{H}+\Omega\right)^{-1} \mathbf{r}\right\} .
$$

Since, the signal vector $\mathbf{r}$ includes the multiple outputs of the same input signal through MR based receiver in relaying phases, to remove redundant coordinates and obtain the solution, we use singular value decomposition.

\section{NUMERICAL RESULTS}

In this section, we present a comprehensive Monte Carlo simulation study for the performance evaluation of the proposed cooperative UWAC system. To explicitly take into account the effects of the relay location, we consider long-term path loss along with the short-term frequency-selective fading. To demonstrate the effects of Doppler distortion. We first use controlled Doppler scaling factors with fixed channel path delays, path gains, transmission power and path loss for all the scenarios. Then, we use the Bellhop software [10] for the calculation of path loss and multipath channel characterization and VirTEX software to introduce the Doppler scaling effects. In this simulation study, we assume that the channel state information is perfectly known.

\section{A. Simulation Results for Different Doppler Scaling Factors}

We consider a cooperative UWAC system with carrier frequency $16 \mathrm{kHz}$ and bandwidth $8 \mathrm{kHz}$. The number of subcarriers is 512 , corresponding to a subcarrier spacing of approximately $15.625 \mathrm{~Hz}$. We consider three different scenarios, which are named Scenario A, B, and C. The channel parameters are listed in Table I.

In Fig. 5, BER performance of multi-resampling and singleresampling compensation over four different Doppler scaling cases including no Doppler case. For the SR receiver, the resampling factor is computed using the average of Doppler scaling factors on each tap weighted by the tap gains. We clearly observe that the performance of the MR receiver is significantly superior to that of the SR receiver. Note that, the difference between the channel parameters is in their Doppler scaling values. Since in Scenarios A and B, the Doppler scaling factors are common to the all paths in all links, no ICI is expected at relay and destination receiver outputs in the broadcasting phase. That is, the MR resampling block will fully compensate the Doppler scaling effects. However, there will be residual interference in relaying phase at the destination due to the addition of the scaled signals by different link specific Doppler scaling factors. The Doppler scaling factor difference between the $\mathrm{S} \rightarrow \mathrm{D}$ and $\mathrm{R} \rightarrow \mathrm{D}$ links for Scenario B is greater than A, hence, the residual ICI will be stronger. This is indeed reflected in Fig. 5 where the proposed system performs better for Scenario A. For Scenario C, we simulate the effect of path specific and link specific Doppler scaling together. In this Scenario, all nodes will end up with residual ICI during both the broadcasting and the relaying phases. In addition, the relay will propagate this interference to the destination. We also include the performance of proposed system with no Doppler scaling, which acts as a lower bound on the error rate performance.

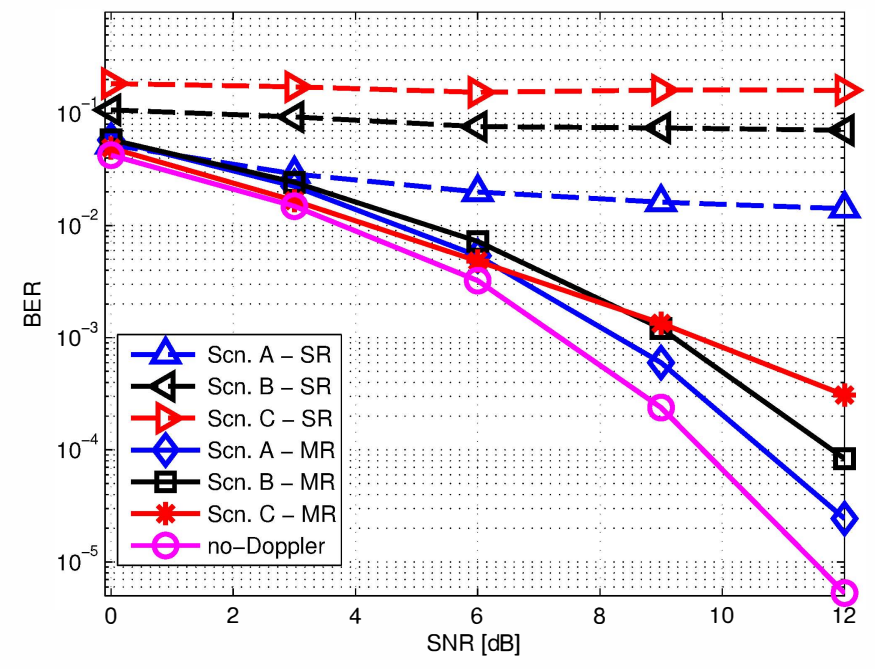

Fig. 5. BER performance of multi-resampling and single-resampling compensation over four different Doppler scaling cases including no Doppler case.

\section{B. Simulation Results for BELLHOP and VirTEX Generated Channels}

In this part of our simulation study, we consider a location in the Arabian Gulf with latitude $26.5^{\circ} \mathrm{N}$ and longitude $52.5^{\circ} \mathrm{E}$. For the given location, environmental factors (such as temperature, salinity and acidity of the water) and the morphology of the sea bottom (such as type of sediment and bathymetry) 
TABLE I

SIMULATION PARAMETERS.

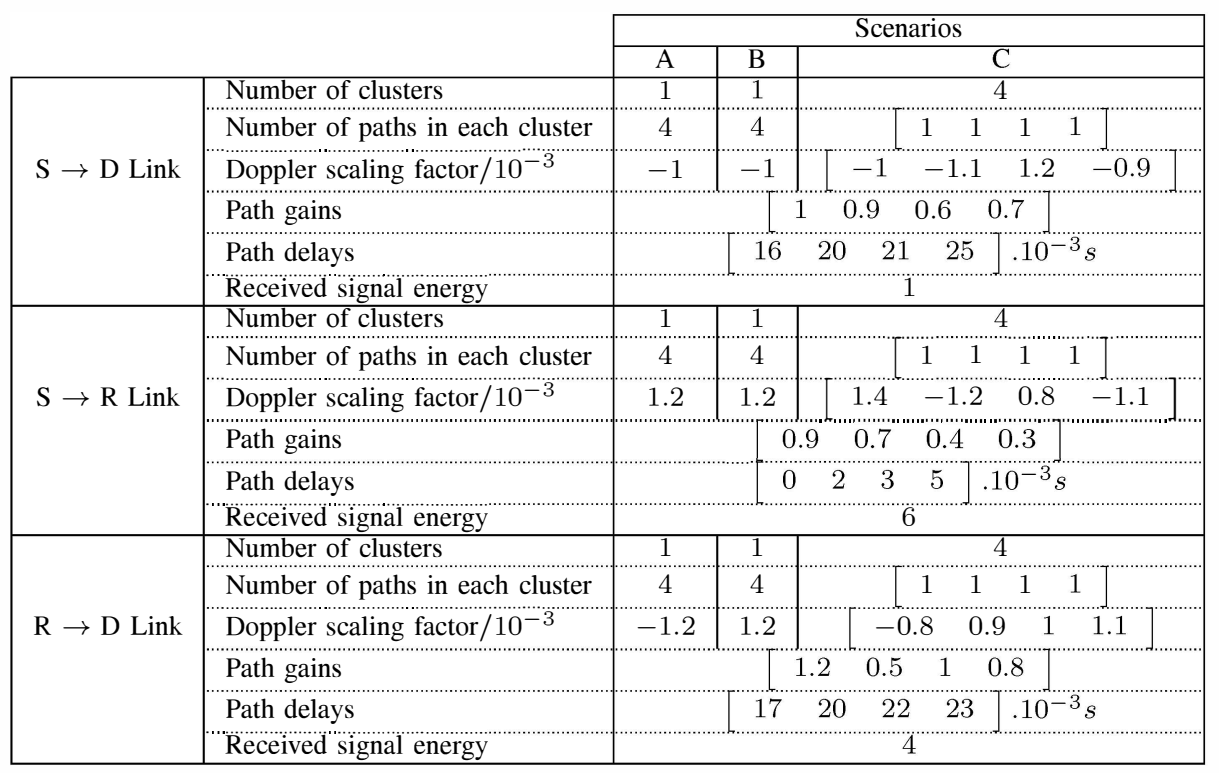

can be found in the databases of US National Oceanic and Atmospheric Administration (NOAA) publicly available at [13]. We consider a cooperative UWAC system with the center
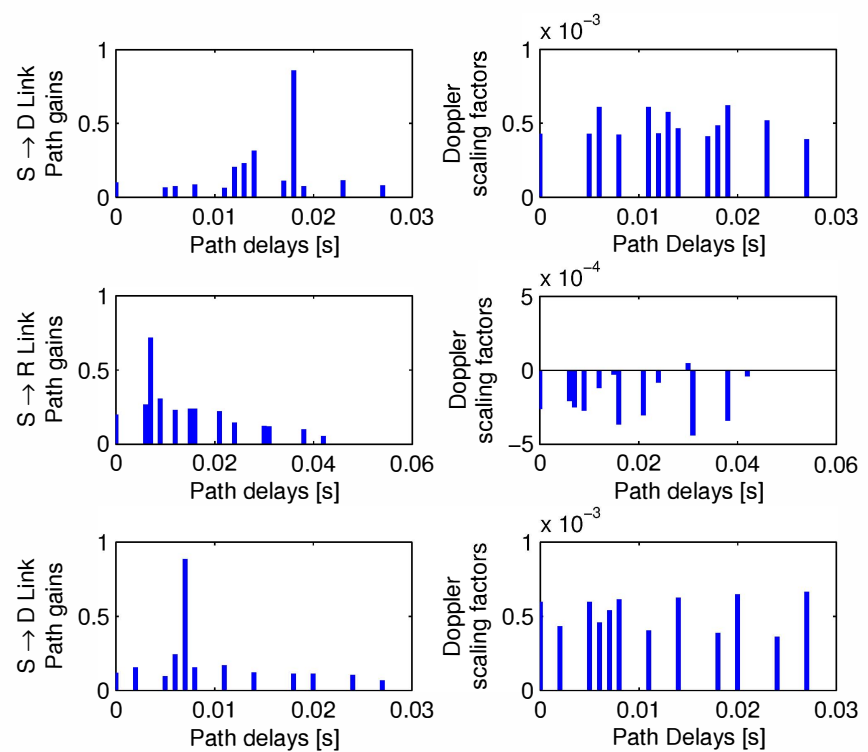

Fig. 6. Each row correspond to path gains versus path delays and Doppler scaling factors versus path delays of the link channels.

frequency of $16 \mathrm{kHz}$ and bandwidth of $1 \mathrm{kHz}$. The number of subcarriers is 256 and therefore the subcarrier spacing is approximately $3.9 \mathrm{~Hz}$. The source and the destination are located $6 \mathrm{~km}$ apart from each other. The relay is placed 2.5 $\mathrm{km}$ away from the source. We assume that the source, relay and destination nodes are located 35, 30, and 40 meters in depths, respectively. The geographical-based data along with the system characteristics such as locations and depths of

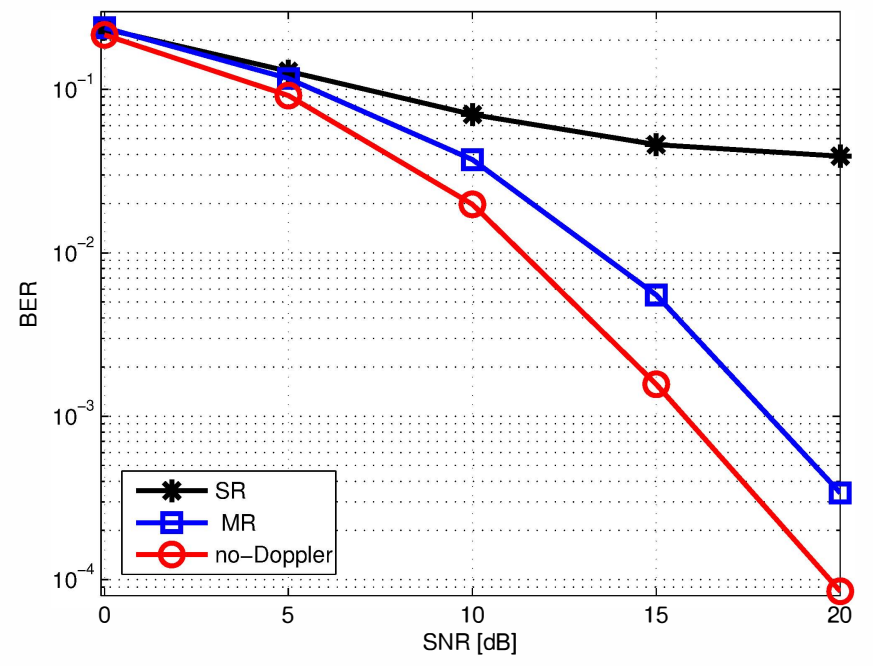

Fig. 7. BER performance of single-resampling compensation, multiresampling compensation and non-Doppler scaling cases.

nodes are given as inputs to the Bellhop software. The software produces arrival data which includes the amplitudes, travel times, angles of arrival, and angles of departure associated with each echo. This yields the deterministic raw channel impulse response which is normalized as in (5) and square of the normalization factor is considered as path loss. To calculate the Doppler scaling factors of the paths in each link, we use BELLHOP output arrival data along with the velocity vector $\left[\begin{array}{ll}\vec{v}_{r} & \vec{v}_{z}\end{array}\right]$ for the nodes, as the input parameters of the VirTEX software, where $\vec{v}_{r}>0$ corresponds to increasing radial distance, and $\vec{v}_{z}>0$ corresponds to motion towards deeper depths. The considered velocity vectors for the source, relay and destination nodes are $\vec{v}_{\mathrm{S}}=\left[\begin{array}{ll}0.8 & 1.6\end{array}\right] \mathrm{m} / \mathrm{s}$, 
$\vec{v}_{\mathrm{R}}=\left[\begin{array}{ll}1.1 & -0.5\end{array}\right] \mathrm{m} / \mathrm{s}$ and $\vec{v}_{\mathrm{D}}=\left[\begin{array}{ll}0 & 0\end{array}\right] \mathrm{m} / \mathrm{s}$, respectively. Resulting channel parameters are shown in Fig. 6. In Fig. 7, the performance results over these channels are provided. It is seen that, MR receiver considerably outperforms the SR receiver. As a benchmark, the performance with fixed terminal nodes (i.e., no Doppler) is also provided.

\section{CONCLUSION}

In this paper, we have proposed a MR receiver design for a cooperative system over motion induced Doppler distorted underwater acoustic channels. We have considered an aggregate channel model that consists of both large-scale path loss and short-term frequency-selective fading. Through the deployment of ray-tracing based Bellhop software and VirTEX software, we have been able to reflect the characteristics of an underwater geographical location and the motion of the nodes on the path loss and end-to-end system performance. We have provided simulation results over different scenarios which exemplify link and path specific Doppler scaling effects.

\section{REFERENCES}

[1] J. Laneman, D. Tse, and G. Wornell, "Cooperative diversity in wireless networks: Efficient protocols and outage behavior," IEEE Transactions on Information Theory, vol. 50, no. 12, pp. 3062-3080, Dec. 2004.

[2] Y. Emre, V. Kandasamy, T. M. Duman, P. Hursky, and S. Roy, "Multi input multi output OFDM for shallow-water UWA communications," in Proceedings of ACOUSTICS 2008, Paris, France, July 2008, pp. 53335338.

[3] S. Mason, C. Berger, S. Zhou, K. Ball, L. Freitag, and P. Willett, "An OFDM Design for Underwater Acoustic Channels with Doppler Spread," in Proc. Digital Signal Processing Workshop and 5th IEEE Signal Processing Education Workshop, Jan. 2009, pp. 138-143.
[4] B. Li, S. Zhou, M. Stojanovic, L. Freitag, and P. Willett, "Multicarrier Communication Over Underwater Acoustic Channels With Nonuniform Doppler Shifts," IEEE Journal of Oceanic Engineering, vol. 33, no. 2, pp. 198-209, Apr. 2008.

[5] S. Yerramalli and U. Mitra, "Optimal Resampling of OFDM Signals for Multiscale-Multilag Underwater Acoustic Channels," IEEE Journal of Oceanic Engineering, vol. 36, no. 1, pp. 126-138, Jan. 2011.

[6] K. Tu, T. M. Duman, M. Stojanovic, and J. G. Proakis, "MultipleResampling Receiver Design for OFDM Over Doppler-Distorted Underwater Acoustic Channels," IEEE Journal of Oceanic Engineering, (online version), 14 pages, Dec. 2012.

[7] K. Tu, D. Fertonani, T. M. Duman, M. Stojanovic, J. G. Proakis, and P. Hursky, "Mitigation of Intercarrier Interference for OFDM Over Time-Varying Underwater Acoustic Channels," IEEE Journal of Oceanic Engineering, vol. 36, no. 2, pp. 156-171, Apr. 2011.

[8] S. Yerramalli and U. Mitra, "Optimal power allocation and Doppler compensation in cooperative underwater networks using OFDM," in IEEE Oceans, Oct. 2009.

[9] K. Tu, T. M. Duman, J. G. Proakis, and M. Stojanovic, "Cooperative MIMO-OFDM communications: Receiver design for Doppler-distorted underwater acoustic channels," in 2010 Conference Record of the Forty Fourth Asilomar Conference on Signals, Systems and Computers. IEEE, Nov. 2010, pp. 1335-1339.

[10] M. Porter, "Bellhop Gaussian beam/finite element beam code," Available in the Acoustics Toolbox at: http://oalib.hlsresearch.com, Last accessed, April 2013.

[11] J. Peterson, M. Porter, and M. Siderius, "Virtual Timeseries EXperiment code," Available at: http://oalib.hlsresearch.com, Last accessed, April 2013.

[12] K. Tu, "Multi-Carrier Communications Over Underwater Acoustic Channels," Ph.D. dissertation, Schl. Electr. Comput. Energy Eng., Arizona State Univ., Tempe, AZ, December 2011.

[13] "U.S. National Oceanic and Atmospheric Administration," Available at: http://www.noaa.gov, Last accessed, 2013. 